\title{
Coupling of single nitrogen-vacancy defect centers in diamond nanocrystals to optical antennas and photonic crystal cavities
}

\author{
Janik Wolters ${ }^{* 1}$, Günter Kewes', Andreas W. Schell', Nils Nüsse², Max Schoengen², Bernd Löchel ${ }^{2}$, \\ Tobias Hanke, Rudolf Bratschitsch ${ }^{3,4}$, Alfred Leitenstorfer ${ }^{3}$, Thomas Aichele', and Oliver Benson ${ }^{1}$ \\ 'Humboldt-Universität zu Berlin, Institut für Physik, Berlin, Germany \\ ${ }^{2}$ Helmholtz-Zentrum Berlin für Materialien und Energie GmbH, Berlin, Germany \\ ${ }^{3}$ Department of Physics and Center for Applied Photonics, Universität Konstanz, Konstanz, Germany \\ ${ }^{4}$ Technische Universität Chemnitz, Institut für Physik, Chemnitz, Germany
}

Keywords diamond nanocrystals, nanomanipulation, optical antennas, photonic crystals, single-photon sources

* Corresponding author: e-mail janik.wolters@physik.hu-berlin.de, Phone: $+49-30-2093-4991$, Fax: +49-30-2093-4718

We demonstrate the abiny to nodity the enission properties and enhares the nteraction strength: or:single-photon eniliors eoupled to rarophotonic structures based on metals and

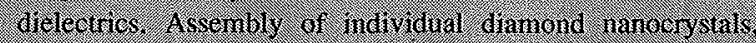

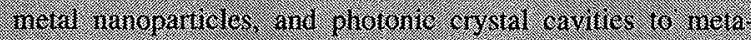
strictures is introdiecd Experinents concening controlle. coupling of singte derect eenters in tangdiamonds to optrea rano.ntennas nade or gold bowhe structures are reviewed. By. phading one and the sanse enutcet at various locations with high prectsion. a riap of decay rate enhancenents was obtained Firthermore, we dernonsuate the fomatron of a hybrid cavity

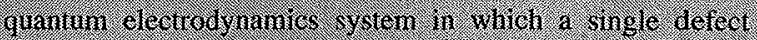

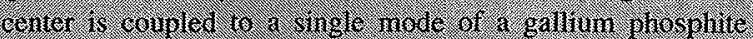
photonic ar stat oavily
1 Introduction In the last decade quantum information processing (QIP) has become one of the major research topics in physics [1]. Here, the main problem is to realize platforms in which complex quantum systems are well isolated from the environment and perfecly controlled at the same time. Many groups all over the world intend to realize such systems based on atoms, ions, or solids [2]. For the latter, most approaches rely purely on semiconductors [3], but recently diamond has emerged as an interesting alternative. In diamond, many optically active defect centers are known and were studied on the single center level. Among those defect centers, the nitrogen-vacancy (NV) center is the most promising for QIP applications [4]. It provides a triplet ground state and is thus capable to encode quantum bits which can be controlled by microwave and read out by optical fields. Electron spin coherence times in the millisecond range were reported [5]. Furthermore, the NV center is intensely used as single-photon source operating at room temperature [6-8].

NV centers occur naturally or can be artificially inserted into the diamond crystal by ion implantation [9].
Combined with lithographic techniques, this allows permanent integration of color centers into microcavities and waveguides $[10,11]$. In contrast, pre-selected diamond nanocrystals containing single defect centers can be coupled to photonic nanostructures to build hybrid quantum systems in a bottom-up approach. With modern nanopositioning techniques in scanning electron microscopy [12, 13] or atomic force microscopy (AFM) [14-16], these diamond nanocrystals can be positioned with a precision of few nanometers.

Several attempts have been pursued to enhance the spontaneous emission (SE) rate of NV centers by coupling them to plasmonic or dielectric resonators: dielectric mircrospheres [17, 18], microtoroids, photonic crystal cavities $[19,20]$ and plasmonic nanoantennas $[21,22]$. In the next sections, we give an overview on our results of coupling single NV centers in diamond nanocrystals to photonic crystal cavities and plasmonic bowtie-antennas.

2 Preparation of single NV centers in diamond nanocrystals In the experiment we use the set-up shown in 


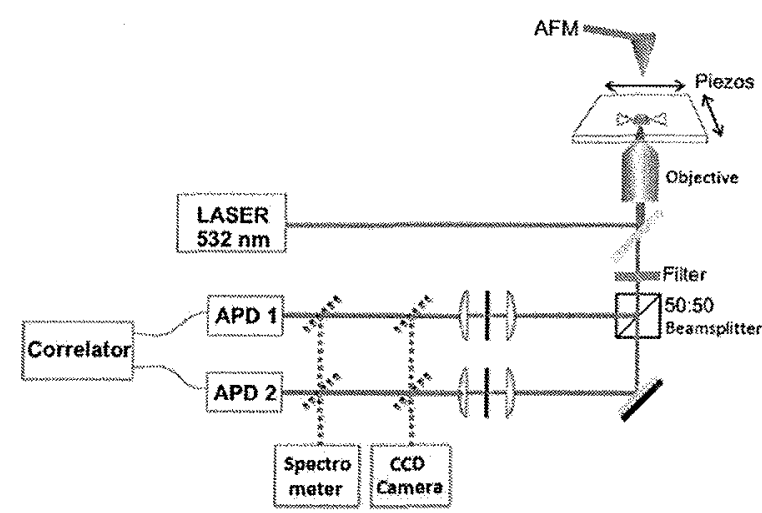

Figure 1 (online color at: www.pss-b.com) Sketch of the optical set-up. The NV centers are excited and their fluorescence is detected through an inverted microscope equipped with a piezo scanning stage. The detected light can be directed to a CCD camera, a spectrometer, or an HBT set-up based on two APDs and time correlation electronics. From top, the sample can be scanned and nanomanipulated with an AFM.

Fig. 1, which consists of a confocal microscope with an AFM (JPK Instruments) on top. The sample is optically excited through the microscope, fluorescence light is collected with the same objective and detected by two avalanche photo diodes (APDs). The APDs together with a beamsplitter form a Hanbury-Brown and Twiss (HBT) set-up. The photon detection times and the trigger signal of the excitation laser were recorded with a photon counting system (Picoquant) in time-tagged time-resolved (TTTR) mode, in which all detection events are stored with $250 \mathrm{ps}$ precision. From this data, the correlation function $g^{(2)}(\tau)=\langle: \hat{I}(t) \hat{I}(t+\tau):\rangle /\langle\hat{I}(t)\rangle^{2}$ and lifetime transients were obtained $(. \ldots)$ indicates normal ordering of the operators). The nanodiamonds were prepared in the following way: first a commercial suspension of $25 \mathrm{~nm}$ sized diamonds (microdiamant) was spin-coated onto a glass cover slip. We estimate that about $1 \%$ of the nanodiamonds host a NV center. Subsequently, these diamonds were precharacterized with a confocal microscope (numerical aperture $\mathrm{NA}=1.35$ ) and a pulsed $532 \mathrm{~nm}$ laser at a repetition rate of $10 \mathrm{MHz}$ and a pulse length of $100 \mathrm{ps}$ (Picoquant LDH-PFA$530)$. Figure $6(\mathrm{~d})$ shows the measured $g^{(2)}(\tau)$ function of a single nanodiamond. For classical light one would expect one peak every $100 \mathrm{~ns}$ representing the repetition rate of the pulsed laser. In contrast, there is no peak at $\tau=0$ ns (i.e., antibunching), showing that the diamond is a single-photon emitter, and thus contains only a single NV center. A typical photoluminescence spectrum of an NV center in a nanodiamond at room-temperature is depicted in Fig. 2. The peak around $639 \mathrm{~nm}$ corresponds to the zero-phonon-line (ZPL) of the negatively charged NV center, the broad band between ca. 600 and $800 \mathrm{~nm}$ to phonon-assisted transitions. After identifying a proper nanodiamond, we used the tip of an AFM to pick it up and transfer it to another substrate containing either plasmonic bowtie antennas or photonic

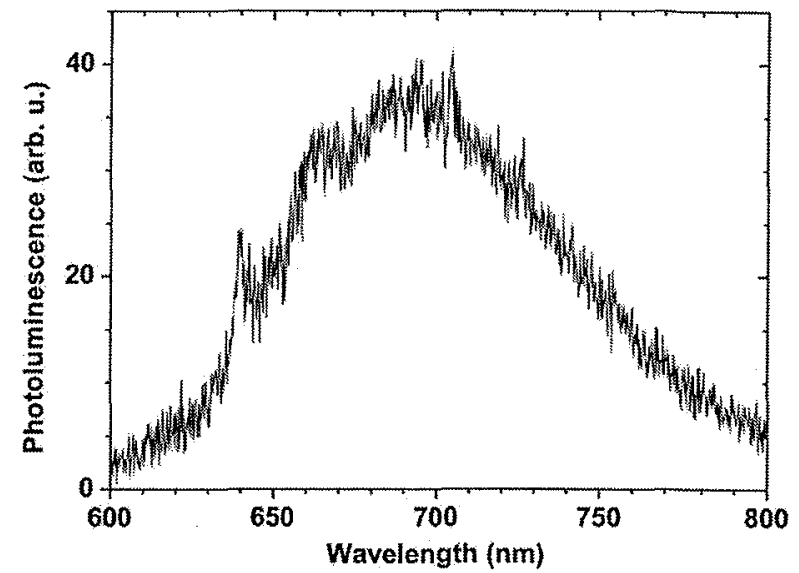

Figure 2 A typical fluorescence spectrum of an NV center in a diamond nanocrystal.

crystal cavities, as described in the following sections. With this technique one can be sure that there is exactly one diamond containing exactly one single NV defect center on the desired position or even on the whole sample. Nanomanipulation with the AFM tip then allowed fine-positioning of the nanocrystal and launching of a single excitation at arbitrary positions near these photonic components.

3 Coupling of single NV centers to plasmonic bowtie antennas In this experiment, the NV center in the nanodiamond is used as a probe for mapping the local electromagnetic environment of a plasmonic nanoantenna. Optical nanoantennas can focus electromagnetic energy in so called hotspots much smaller than the optical wavelength and thus lead to higher excitation efficiency of photon emitters. Additionally, the local density of optical states (LDOS) is increased due to the presence of surface plasmonic modes in the antenna structure [23]. This leads to higher radiative emission rates $\Gamma_{\mathrm{r}}$, as well as enhanced nonradiative rates $\Gamma_{\mathrm{nr}}$ due to Ohmic damping of surface plasmons in the metal. With molecules as emitters, enhancement of the fuorescence signal by over 1000 was recently reported [24]. As a local probe for the enhancement of excitation and decay rates, an almost point-like light source with high stability during the mapping is needed. In addition, the probe has to be positioned with nanometer precision. These key requirements are perfectly provided by single NV centers in nanodiamonds, especially when combined with an AFM nanomanipulation technique.

The plasmonic nanostructure we want to probe is a bowtie nanoantenna fabricated out of gold via electron-beam lithography [22]. Simulations of such antennas predict a high electromagnetic field intensity in the antenna gap (where the tips of the two triangles face each other) which correspond to a high LDOS.

As described before, using the tip of an AFM, the precharacterized nanodiamond with a single NV center was 


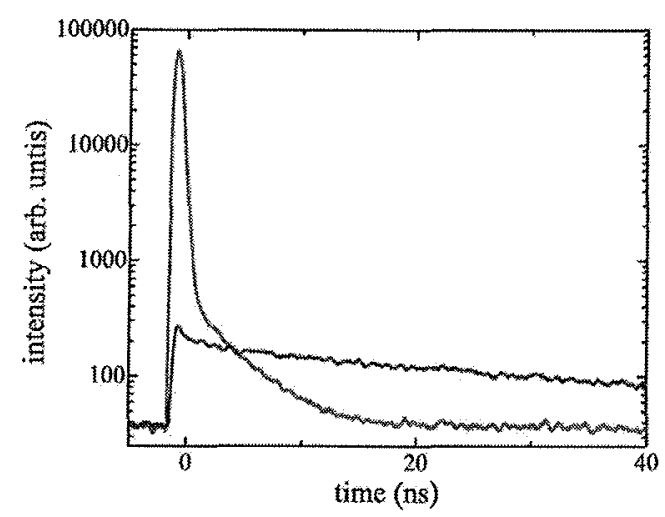

Figure 3 (online color at: www.pss-b.com) Liftime curves (log plots) of a single NV center in a nanodiamond at different positions on the antenna sample. Green curve: the nanodiamond is positioned several micrometers away from the antenna. Blue curve: the nanodiamond is placed close to the antenna gap. At this position a maximum lifetime shortening was observed. The fast decaying signal $(t<2 \mathrm{~ns})$ stems from gold fluorescence, the slower component $(t>2 \mathrm{~ns})$ from the NV center.

transferred to the nanoantenna sample and placed at various different positions relative to the nanoantenna. The size of the chosen nanodiamond was only $15 \mathrm{~nm}$. On each position, a fluorescence decay curve was taken. Figure 3 shows two different lifetime measurements for a nanodiamond several micrometers away from the plasmonic structure (blue) and close to the antenna gap (green). The blue curve shows a nearly mono-exponential decay revealing the lifetime of the uncoupled NV center ( $\tau_{\mathrm{uc}}=38.4 \mathrm{~ns}$ ). The green curve however shows a fast decay component clearly below $1 \mathrm{~ns}$ (the resolution is limited by the instrument response time) stemming from gold fluorescence processes, followed by the

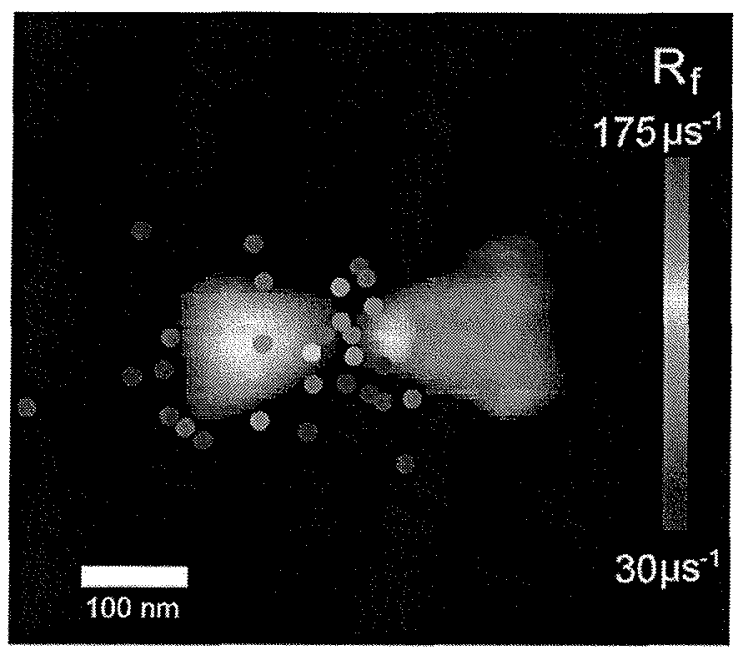

Figure 4 (online color at: www.pss-b.com) Map showing the decay rate $R_{f}$ (colorscale) of an NV center in the diamond nanocrystal at different positions (colored dots) around the bowtie nanoantenna (AFM scan). signal from the coupled NV center. The NV center lifetime was extracted by fitting a monoexponential decay-curve to the data starting about $2 \mathrm{~ns}$ after excitation.

We placed and characterized the nanodiamond at various other positions around the nanoantenna. In doing so, we derived a map of decay rates (the inverse of the lifetime) of the excited state of the NV center close to the plasmonic nanoantenna as shown in Fig. 4. The underlay shows the AFM topography image of the nanoantenna, while the points indicate the position of the nanodiamond and their color the measured decay rate. The maximum observed decay rate occured inside the gap (lifetime $\tau_{c}=3.6 \mathrm{~ns}$ ), where it was increased by a factor of 10.7 compared to its decay rate several micrometers away from the nanoantenna.

To distinguish between enhancement effects of radiative and non-radiative rates, saturation measurements have to be performed [25]. Unfortunatly, in our experiments, the gold nanoantennas heated up when exciting the sample with laser light and started to melt for laser powers above $80 \mu \mathrm{W}$. Figure 5 shows several AFM scans after excitation with different laser powers. One can clearly see that the surface roughness is reduced while the gap is increased. Due to this, no complete saturation curve could be measured yet. In previous experiments with gold nanospheres as nanoantennas, however, a radiative rate enhancement up to 8.9 was observed [21]. Nevertheless, the effect of gold melting opens an easily accessible way to tune the resonance of an gold nanoantenna since it depends on both the shape and the antenna gap.

To ensure that the single-photon character of the NV center remains after coupling to the nanoantenna, a time correlated single photon counting measurement was
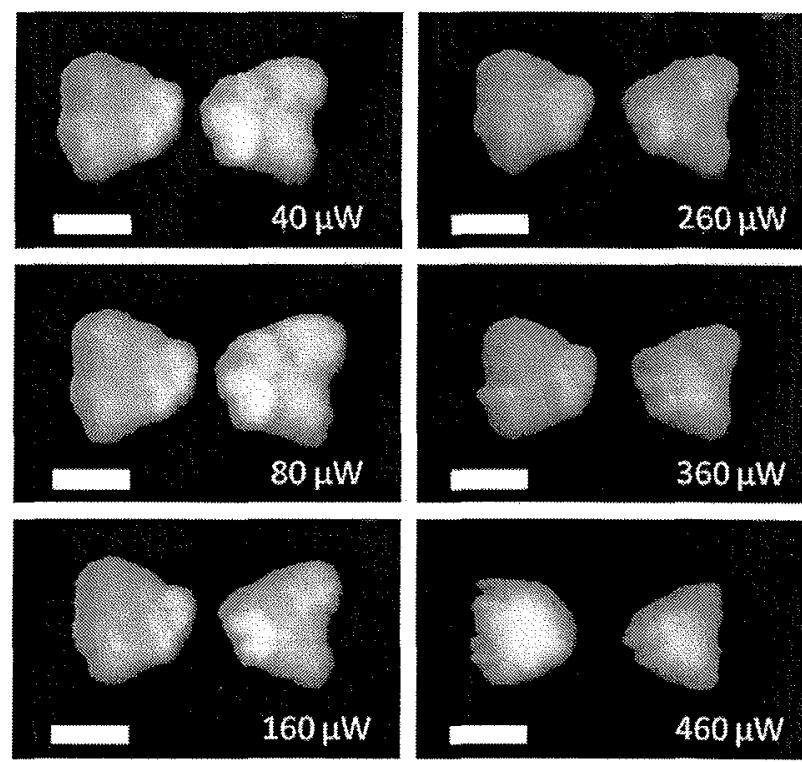

Figure 5 (online color at: www.pss-b.com) AFM scans of a gold nanoantenna after illumination with a $532 \mathrm{~nm}$ pulsed laser for I min at different powers. Above $80 \mu \mathrm{W}$, the gold starts to melt causing a deformation of the antenna and an increase of the antenna gap. 

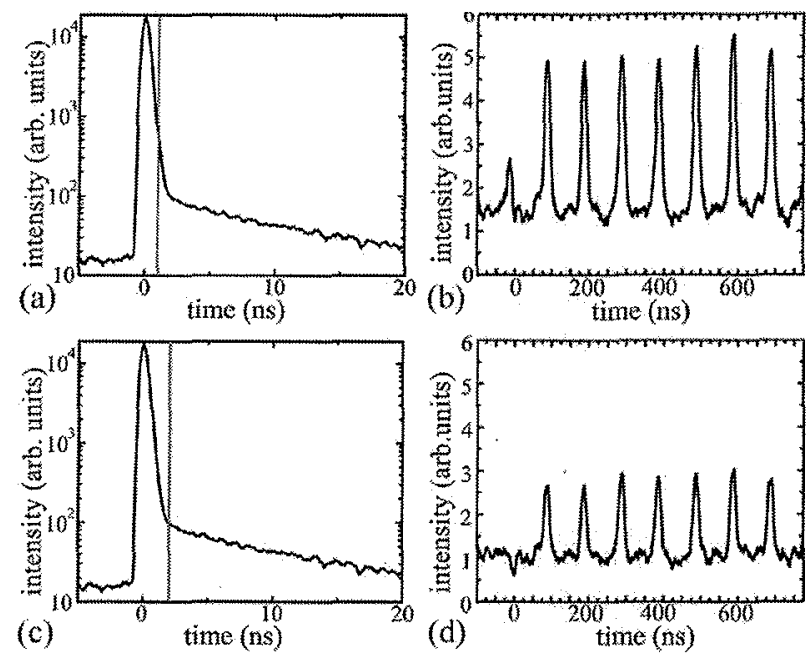

Figure 6 (online color at: www.pss-b.com) Data obtained from time tagged correlation measurements. (a) and (c) show the fluorescence decay curve. Only detection events occuring later than the gating time (marked by the vertical red lines) were used when reconstructing the autocorrelation measurements in (b) and (d), respectively. The gating times were $\Delta t=1 \mathrm{~ns}$ for (b), and $\Delta t=2 \mathrm{~ns}$ for (c), respectively.

performed in the TTTR mode. The exact knowledge of the detection times and of the trigger signals from the pulsed laser diode allowed a temporal separation of fluorescence decaying at different time scales. In this way the uncorrelated photon emission processes of the fluorescing gold structures can be eliminated during a post-process where all counts which occured during a short time after the trigger signal of the pulsed laser where ignored. Thus, only light belonging to the longer living excited state of the NV center contributes to the autocorrelation measurement. Figure 6 shows this evaluation for two different gating windows. The increased correlation peak at $t=0$ of Fig. 6(b) shows that multi-photon events appear below $\approx 2 \mathrm{~ns}$ after the laser pulse, while at larger times the photon statistics is completely anti-bunched, i.e., of single-photon character, Fig. 6(d). This demonstrates that indeed a single quantum emitter, here a single NV center, was utilized to map the local fluorescence enhancement around a plasmonic antenna (Fig. 4).

4 Coupling of single NV centers to photonic crystal cavities In optical cavities, the $\mathrm{SE}$ rate is enhanced by the Purcell effect. For a perfect matching of the emitter's dipole with the cavity field, the SE rate enhancement into the cavity mode is described by the Purcell factor

$$
F=\frac{3}{4 \pi^{2}}\left(\frac{\lambda_{c}}{n}\right)^{3} \frac{Q}{V_{\mathrm{eff}}}
$$

Here, $\lambda_{c} / n$ is the wavelength at the cavity resonance in the dielectric, $Q$ is the quality factor of the cavity resonance ( $Q$-factor) and $V_{\text {eff }}$ is the effective mode volume. Increasing the SE rate into the ZPL allows the generation of a large number of indistinguishable single photons [26] needed for example in linear optics quantum computation [27].

For the coupling of individual quantum emitters, photonic crystal cavities have several advantages. They can be designed and manufactured with ultra high quality factors [28], while providing ultra small mode volumina in the order of the qubic wavelength. Both are figures of merit for obtaining a large Purcell factor. Furthermore, waveguides can be easily incorporated into photonic crystals, thus complex chipscale systems of coupled cavities and waveguides can be produced [29]. In the following, we present results on the coupling of the ZPL of an NV center in a nanodiamond to a single mode of a photonic crystal cavity. This is a crucial step towards integrated diamond-based quantum technology.

We produced a free-standing photonic crystal slab (lattice constant $200 \mathrm{~nm}$ ) with a cavity. We use a so-called L3 cavity, which is formed by three missing holes (see Fig. 7). The structure is designed using finite-difference time-domain (FDTD) simulations (Lumerical) and optimized to have the fundamental mode at $640 \mathrm{~nm}$. This value is

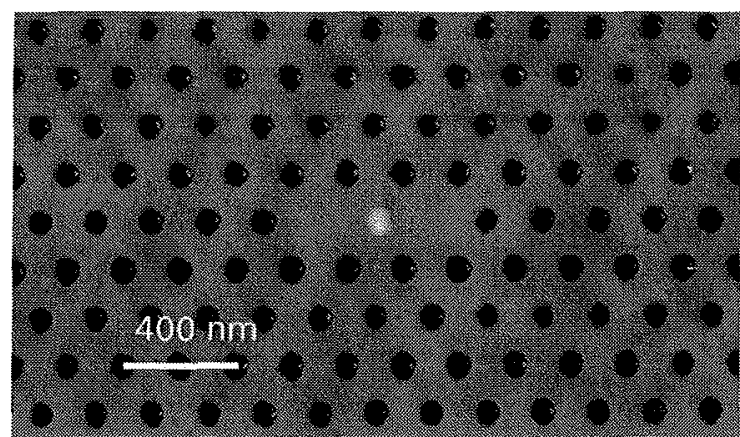

(a)

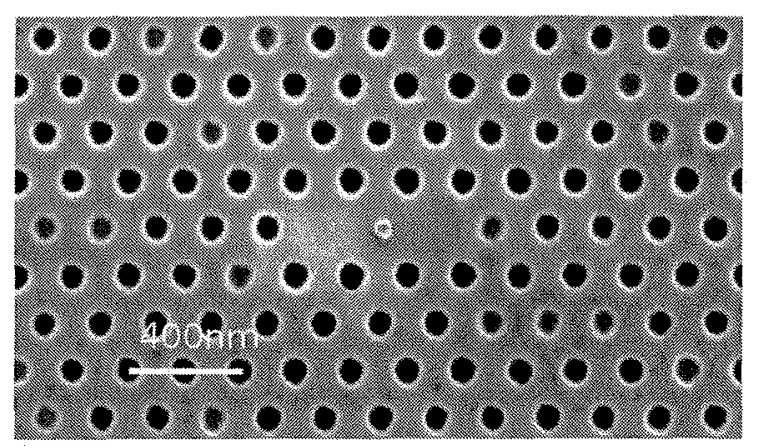

(b)

Figure 7 (online color at: www.pss-b.com) (a) AFM image of the photonic crystal cavity with a single nanodiamond. It is well placed in the center of the cavity, where the electric field distribution of the cavity mode has its maximum. (b) The corresponding scanning electron microscope image of the same region. 
slightly above the ZPL of the NV center. Numerical simulations predict a mode volume of $V_{\text {eff }} \approx 0.75(\lambda / n)^{3}$ for the fundamental mode. We fabricated these structures from a $70 \mathrm{~nm}$ thick heteroepitaxial gallium phosphide (GaP) layer [30], deposited on a Si(100) substrate. The wafers were structured by electron beam lithography and subsequently dry-etched.

To characterize the structures, we use a home-built micro photoluminescence set-up with a numerical aperture of 0.9 .

The fluorescence light from the bare cavities under strong excitation with a $532 \mathrm{~nm}$ laser $(300 \mu \mathrm{W})$ was analyzed with a $500 \mathrm{~mm}$ spectrometer. As the intrinsic fluorescence is strongly enhanced at the cavity resonance, the resonances appear as peaks in the spectra. All the fabricated cavities show resonances around $642 \mathrm{~nm}$ with a typical deviation of $\pm 2 \mathrm{~nm}$ and $Q$-factors of around 1000 (Fig. 8).

In order to match the cavity resonances to the ZPL of the NV centers, which occurs at about $639 \mathrm{~nm}$, a fine-tuning can be performed by local heating. Therefore a blue $(407 \mathrm{~nm})$ laser beam is focused onto an individual photonic crystal membrane. As the photon energy is above the bandgap of $\mathrm{GaP}$, the laser is almost completely absorbed within the membrane and heats the structure locally. It is widely assumed, that the material oxidizes [31] and changes the index of refraction, i.e., the material gets optically thinner. By this procedure, the cavity resonances can be irreversibly tuned by up to $4 \mathrm{~nm}$ to the blue (see Fig. 8). Surprisingly, the wavelength shift does not depend on the heating time but on the heating power. With about $120 \mu \mathrm{W}$ of heating power, the resonance is shifted by about $0.1 \mathrm{~nm}$, while it is shifted by about $3 \mathrm{~nm}$ with $700 \mu \mathrm{W}$. At tuning ranges below $2 \mathrm{~nm}$, the Q-factor remains roughly unaffected (Fig. 9). It is even possible to slightly increase the Q-factor. Only at highest tuning ranges, the cavity degenerates and the Q-factor is significantly lowered.

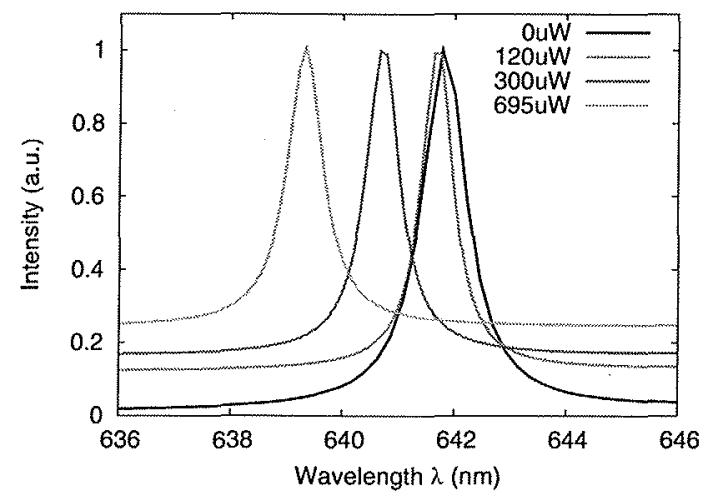

Figure 8 (online color at: www.pss-b.com) Part of the intrinsic fluorescence spectra of a GaP photonic crystal cavity around $640 \mathrm{~nm}$. The fundamental cavity resonance can be permanently tuned by local heating with a focused $407 \mathrm{~nm}$ laser. Interestingly, the lineshift depends on the heating power, rather than on the heating time ( $30 \mathrm{~s}$ in all cases). The total tuning range is up to $3 \mathrm{~nm}$.

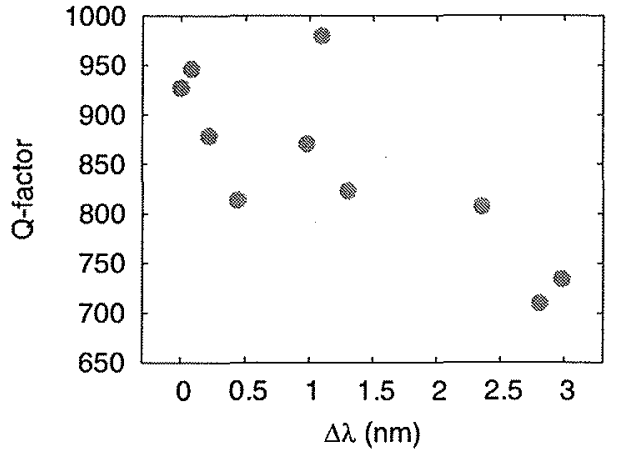

Figure 9 (online color at: www.pss-b.com) When tuning the cavity resonance, the quality factor remains almost unaffected. Sometimes, even an improvement is possible. Only at larger tuning shifts (about $2 \mathrm{~nm}$ ), a degradation of the cavity quality factor is measureable.

With this technique, it is possible to tune an individual cavity resonance exactly to the ZPL of a preselected NV center, as shown in Fig. 10.

After tuning, a preselected nanodiamond is picked up from a cover slip with the AFM using the pick-and-place method. As it is not possible to place the diamond directly in the cavity, it is preliminarily placed on the GaP surface, close to the photonic crystal cavities [16]. In a final step, the diamond is picked up again and this time placed exactly in the center of the cavity, as shown in Fig. 7. The exact positioning of the diamond is crucial, as the field strength of the cavity mode decays rapidly outside the center. Furthermore, it is important to place the NV center as close as possible to the surface, as the cavity mode decays exponentially outside the slab [19]. We used a diamond with a height of about $35 \mathrm{~nm}$, thus the NV center is $<35 \mathrm{~nm}$ above the surface, allowing for a high coupling efficiency.

Figure 11 shows the measured spectra of the NV center inside and outside of the cavity. The enhancement of the ZPL emission at $639.5 \mathrm{~nm}$ by the Purcell effect is striking! For the

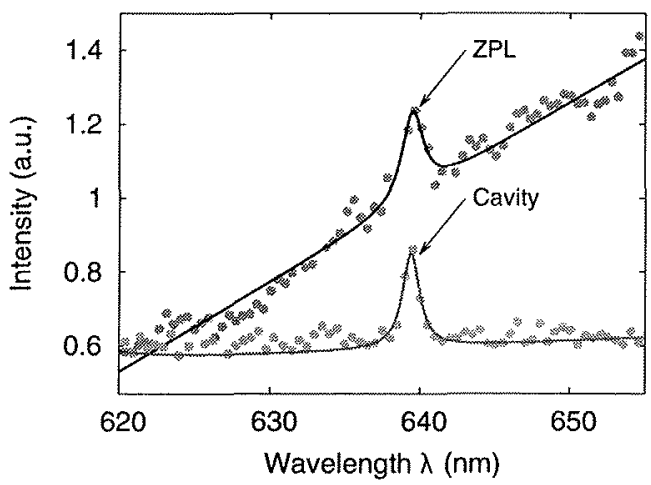

Figure 10 (online color at: www.pss-b.com) Measured spectra (red and green points, respectively) and the correspondig fits (black and blue line, respectively) to the ZPL of the preselected NV center and the tuned cavity. After the tuning the cavity resonance matches the ZPL of the NV center almost perfectly (from Ref. [20]). 


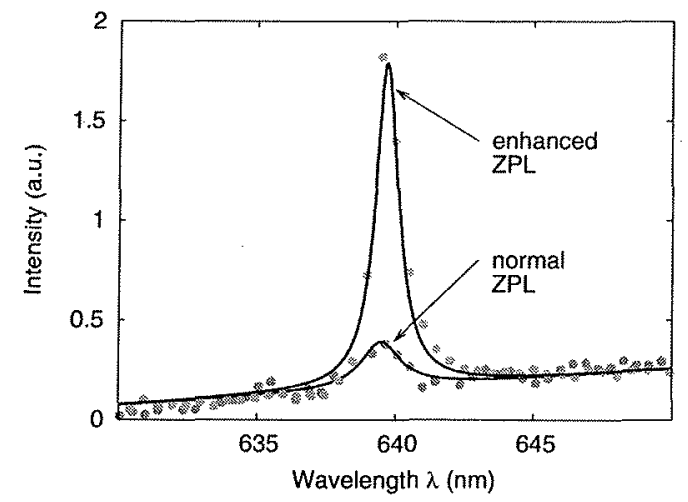

Figure 11 (online color at: www.pss-b.com) The ZPL of the NV center in and outside of the photonic crystal cavity. The enhancement is tremendous.

green curve in Fig. 11, we subtracted the bare cavity background, thus the displayed intensity stems only from the NV center. Care has to be taken when comparing the optical properties of a bare defect center and one coupled to a photonic structure. Any change in the dielectric environment also influences the optical properties (emission rate and pattern) [19]. Since only a small fraction of the emission spectrum is enhanced by the cavity, it is not possible to estimate the coupling efficency by measuring the small change of the overall lifetime.

To calculate the pure effect of the cavity resonance, both spectra are normalized with respect to the NV emission left and right of the ZPL. Then, we calculated the spectrally resolved SE enhancement due to the cavity $F^{*}=12.1$. Equation (1) predicts an enhancement of $F=60$ with $Q=600$ and $V=0.75\left(\lambda_{c} / n\right)^{3}$. The difference between experimental and theoretical value is caused by non-perfect matching of the emission profile of the NV center and the cavity mode: The dipole is neither perfectly oriented nor placed direcly in the field maximum, which is in the center of the slab.

As a further proof, that the luminescence stems from the NV center, an autocorrelation measurement was performed. The measured autocorrelation function $g^{(2)}(\tau)$ clearly revealed a non-classical behavior, which can originate only from the NV center.

For further improvement of the emission properties, cavities with higher Q-factor can be used and the experiment can be performed at cryogenic temperatures. Here, the spectral width of ZPL can be lifetime-limited [32] and about $3 \%$ of the total radiation are emitted into the ZPL. Under these assumptions, using a cavity with a $Q$-factor of 600 about $30 \%$ of the radiation will be channeled into the cavity mode. This means an increase of the Debye-Waller factor by about 10 , allowing for more advanced quantum-optical experiments.

5 Conclusion In conclusion, we demonstrate the deterministic nanopositioning and coupling of single NV centers in nanodiamonds to different nanophotonic components. By bringing the emitter close to an optical nanoantenna, information about the local electromagnetic field and the local density of states could be probed. Such mapping of near-field properties of nanostructures is crucial for optimization of designs or quality control. In a second experiment, the zero phonon line of a single NV center in a nanodiamond was coupled to a photonic crystal cavity. This is a major step towards the realization of integrated quantum optical devices. With the presented pick-and-place technique and the selective cavity tuning, even more complex systems, involving two cavities and emitters [33] can be assembled in a controlled way. Simple quantum gates, integrated on a single photonic crystal chip, are within reach.

Acknowledgements We thank $H$. Döscher and from Helmholtz-Zentrum Berlin for providing GaP wafers. This work was supported by DFG (BE2224/9 and AI92/3) and BMBF (KEPHOSI). J. Wolters acknowledges funding by the state of Berlin (NaFöG).

\section{References}

[1] T. D. Ladd, F. Jelezko, R. Laflamme, Y. Nakanura, C. Monroe, and J. L. O'Brien, Nature 320, 1601 (2010).

[2] J. L. O'Brien, A. Furusawa, and J. Vuckovic, Nature Photon. 3, 687 (2009).

[3] I. Fushman, D. Englund, A. Faraon, N. Stoltz, P. Petroff, and J. Vuckovic, Science 320, 5877 (2008).

[4] F. Jelezko and J. Wrachtrup, Phys. Status Solidi A 203, 21 (2006).

[5] G. Balasubramanian, P. Neumann, D. Twitchen, M. Markham, R. Kolesov, N. Mizuochi, J. Isoya, J. Achard, J. Beck, J. Tissler, V. Jacques, P. R. Hemmer, F. Jelezko, and J. Wrachtrup, Nature Mater. 8, 383 (2009).

[6] Ch. Kurtsiefer, S. Mayer, P. Zarda, and H. Weinfurter, Phys. Rev. Lett. 85, 290 (2000).

[7] R. Brouri, A. Beveratos, J.-P. Poizat, and P. Grangier, Opt. Lett. 17, $1294(2000)$

[8] T. Schröder, A. Schell, G. Kewes, T. Aichele, and B. Benson, Nano Lett. 11, 198 (2011).

[9] J. Meijer, S. Pezzagna, T. Vogel, B. Burchard, H. Bukow, I. Rangelow, Y. Sarov, H. Wiggers, I. Plmel, F. Jelezko, J. Wrachtrup, F. Schmidt-Kaler, W. Schnitzler, and K. Singer, Appl. Phys. A 91, 567 (2008).

[10] K.-M. C. Fu, C. Santori, P. E. Barclay, I. Aharonovich, S. Prawer, N. Meyer, A. M. Holm, and R. G. Beausoleil, Appl. Phys. Lett. 93, 234107 (2008).

[11] T. M. Babinec, J. T. Choy, K. J. M. Smith, M. Khan, and M. Loncar, J. Vac. Sci. Technol. B 29, 010601 (2011).

[12] T. van der Sar, E. C. Heeres, G. M. Dmochowski, G. de Lange, L. Robledo, T. H. Oosterkamp, and R. Hanson, Appl. Phys. Lett. 94, 173104 (2009).

[13] E. Ampem-Lassen, D. A. Simpson, B. C. Gibson, S. Trpkovski, F. M. Hossain, S. T. Huntington, K. Ganesan, L. C. Hollenberg, and S. Prawer, Opt. Express 17, 11287 (2009).

[14] M. Barth, N. Nüsse, B. Löchel, and O. Benson, Opt. Lett. 34, $1108(2009)$.

[15] A. Huck, S. Kumar, A. Shakoor, and U. L. Andersen, Phys. Rev. Lett. 106, 096801 (2011). 
[16] A. W. Schell, G. Kewes, T. Schröder, J. Wolters, T. Aichele, and O. Benson, Rev. Sci. Instrum. 82, 073709 (2011).

[17] Y.-S. Park, A. K. Cook, and H. Wang, Nano Lett. 6, 2075 (2006).

[18] S. Schietinger, T. Schröder, and O. Benson, Nano Lett. 8, 3911 (2008).

[19] D. Englund, B. Shields, K. Rivoire, F. Hatami, J. Vuckovic, H. Park, and M. D. Lukin, Nano Lett. 10, 3922 (2010).

[20] J. Wolters, A. W. Schell, G. Kewes, N. Nüsse, M. Schoengen, H. Döscher, T. Hannappel, B. Löchel, M. Barth, and O. Benson, Appl. Phys. Lett. 97, 141108 (2010).

[21] S. Schietinger, M. Barth, T. Aichele, and O. Benson, Nano Lett. 9, 1964 (2009).

[22] A. W. Schell, G. Kewes, T. Hanke, A. Leitenstorfer, R. Bratschitsch, O. Benson, and T. Aichele, Opt. Express 19, 7914 (2011).

[23] L. Novotny and B. Hecht, Principles of Nano-Optics (Cambridge Univ. Press, Cambridge, 2006).

[24] A. Kinkhabwala, Z. Yu, S. Fan, Y. Avlasevich, K. Müllen, and W. E. Moerner, Nature Photon. 3, 654 (2009).
[25] I. Gregor, D. Patra, and J. Enderlein, Chem. Phys. Chem. 6, 164 (2005).

[26] C.-H. Su, A. D. Greentree, and L. C. L. Hollenberg, Opt. Express 16, 12 (2008).

[27] E. Knill, R. Laflamme, and G. J. Milburn, Nature 409, 46 (2001).

[28] B.-S. Song, S. Noda, T. Asano, and Y. Akahane, Nature Mater. 1, 468 (2005).

[29] M. Notomi, E. Kuramochi, and T. Tanabe, Nature Photon. 2 , 741 (2008).

[30] H. Döscher, T. Hannappel, B. Kunert, A. Beyer, K. Volz, and W. Stolz, Appl. Phys. Lett. 93, 172110 (2008).

[31] H. S. Lee, S. Kiravittaya, S. Kumar, J. D. Plumhof, L. Balet, L. H. Li, M. Francardi, A. Gerardino, A. Fiore, A. Rastelli, and O. G. Schmidt, Appl. Phys. Lett. 95, 191109 (2009).

[32] Y. Shen, T. M. Sweeney, and H. Wang, Phys. Rev. B 77, 033201 (2008).

[33] C.-H. Su, A. D. Greentree, W. J. Munro, K. Nemoto, and L. C. L. Hollenberg, Phys. Rev. A 78, 062336 (2008). 